\title{
Good Bug Hunting: Inspiring and Motivating Software Testing Novices
}

\author{
Natalia Silvis-Cividjian \\ Vrije Universiteit (VU) \\ Amsterdam, The Netherlands \\ n.silvis-cividjian@vu.nl
}

\author{
Marc Went \\ Robert Jansma \\ Vrije Universiteit (VU) \\ Amsterdam, The Netherlands
}

\author{
Viktor Bonev \\ Emil Apostolov \\ Vrije Universiteit (VU) \\ Amsterdam, The Netherlands
}

\begin{abstract}
Although software testing is crucial in safeguarding our rapidly digitizing society against accidental and malefic threats, students often perceive it as an unattractive activity when compared to design or coding. A more abundant exposure to bugs and their impact can improve the situation. To test this radically new hypothesis, we developed VU-BugZoo, an interactive software testing learning platform. Built upon a repository of fault-seeded executables, the platform engages students and teachers in a dynamic bug-hunting experience. What counts the most in this game is the quality of the test strategy and fault diagnosis, rather than just the quantity of devised test cases. In this paper, we describe the VU-BugZoo web-application, developed using a stack of Vue.js and Python Flask, running in Docker containers. Next, we report on its first deployment in the midst of the COVID-19 pandemic, for a remote assessment of 50 graduate CS students enrolled in an introductory software testing course. Students' evaluations show that for novices, a $100 \%$-bug-guarantee, end-to-end testing challenge (1) makes learning more effective, (2) offers an adequate assessment instrument and (3) adds more excitement to an otherwise uninspiring chore. More research is needed to investigate the potential of our bug-centric approach to contribute to the hard mission of boosting students' interest in a testing career. We are currently extending the corpus of bugs with more fascinating and representative examples, and investigating code animation, automated grading and formative feedback generation. We intend to make the platform available for other software testing courses in academia and industry.
\end{abstract}

\section{CCS CONCEPTS}

- Software and its engineering $\rightarrow$ Software testing and debugging.

\section{KEYWORDS}

software testing education; fault injection; COVID-19 pandemic; remote assessment

\section{ACM Reference Format:}

Natalia Silvis-Cividjian, Marc Went, Robert Jansma, Viktor Bonev, and Emil Apostolov. 2021. Good Bug Hunting: Inspiring and Motivating Software Testing Novices. In 26th ACM Conference on Innovation and Technology in

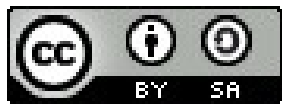

This work is licensed under a Creative Commons Attribution-ShareAlike International 4.0 License.

ITiCSE 2021, June 26-fuly 1, 2021, Virtual Event, Germany.

(C) 2021 Copyright held by the owner/author(s).

ACM ISBN 978-1-4503-8214-4/21/06.

https://doi.org/10.1145/3430665.3456330
Computer Science Education V. 1 (ITiCSE 2021), June 26-fuly 1, 2021, Virtual Event, Germany. ACM, New York, NY, USA, 7 pages. https://doi.org/10.1145/ 3430665.3456330

\section{INTRODUCTION}

Software testing is the process of checking that a software product, usually code, behaves according to its specifications and/or user intentions [33]. Static testing performs this check without running the code, through reviews or inspections. Dynamic testing executes the code by feeding it with inputs and comparing each result to the expected, specified output. If the two are equal, then the test case is declared as passed; otherwise, the test is declared as failed, indicating the presence of a defect in code, also called fault, or bug. History learns that simple bugs can have a huge impact. Examples are a missing semicolon that disrupted the AT \& T telephone network [8], or a race condition in the Therac-25 radiotherapy machine that caused several fatal overexposure incidents [16], to culminate with an omission to implement sensor data sanity checks that contributed to the recent Boeing 737 MAX accidents [23]. It goes without saying that software testers must uncover potentially dangerous bugs as soon as possible, before the product is released to its end-users. The problem is that an exhaustive testing, targeting for all possible inputs, requires a massive amount of time, even for a few lines of code. Therefore, the art of software testing stays in the smart choice of efficient, no-nonsense test inputs. Many test generation techniques exist for this purpose, recommended by textbooks and safety standards. Because there is no silver-bullet test generation technique good for all types of bugs, a test strategy is needed that combines different test techniques with the goal of detecting the highest number of faults in the shortest time. Unfortunately, despite their importance, sound test strategies are rare, remaining the exclusive privilege of mature organizations. The test process ends when the completion criteria are met. The result is a test report, containing all the relevant test cases, including their inputs and expected outputs, and information on whether a test case passed or failed [34]. Often, additional information is also provided, such as the test strategy, a description of the test environment, a description of the abnormal behaviour, and very rarely, tips about what or where the bug might be.

We have been teaching a dedicated course on software testing for more than ten years already. Yearly, around 50 computer science graduate students enroll in the course. Mainly based on the Mathur's, Pezze \& Young's and Copeland's textbooks [7, 18, 28], the course features a series of lectures and invited talks, a few group assignments that test "flawless-by-design" software products, and a closed-book written exam. The design of the course is the reflection of one's belief that a systems-centric approach with an emphasis 
Table 1: Excerpt from students evaluations collected in 2018 $(\mathrm{N}=17)$ and $2019(\mathrm{~N}=14)$, before the intervention.

\begin{tabular}{|l|c|c|c|c|c|}
\hline Question & - & +- & + & $\mathrm{N}$ & Agree \\
\hline Interesting course & 4 & 6 & 21 & 31 & $67 \%$ \\
\hline $\begin{array}{l}\text { Relevance to the pro- } \\
\text { gramme was clear }\end{array}$ & 3 & 4 & 24 & 31 & $76 \%$ \\
\hline $\begin{array}{l}\text { I would like working in the } \\
\text { software testing field }\end{array}$ & 7 & 11 & 10 & 28 & $35 \%$ \\
\hline
\end{tabular}

on bugs and their catastrophic impact, is a powerful tool to educate responsible and enthusiastic testers. The course and its innovative elements are described in detail in [30].

Although the course was always positively received, every year students' evaluations showed us the same, paradoxical disbalance between the high percentage of students who liked the course (around $72 \%$ ) and the much lower percentage of students keen to pursue a career in testing (around 35\%). This contrast, illustrated in Table 1, corroborates with other reports, signaling that software testing is neither a popular course, nor career choice, $[4,5,9,10$, 17, 29, 37]. The reported percentages of students interested in a testing job elsewhere is usually low, varying from $7 \%$ to $40 \%$, with an average situated around $20 \%$. Obviously, this feels like a bitter defeat for us educators, especially now, when enthusiastic testers are highly needed to safeguard our digital society from all kinds of threats. So, what exactly scares our students and refrains them from embracing a career in software testing? We analyzed the situation and inferred two possible root-causes.

(1) Designing test cases without executing them is not motivating. In our opinion, one of the problems is that we mainly teach students how to design test cases based on hypothetical requirements or small code snippets, with no executable in place. A classical assignment in an introductory software testing course usually sounds like: "Generate test cases for requirement X using technique Y". The learning goal in this approach is to develop "a fine nose" on which test technique to choose given a certain requirement specification. Which is of course good, but not good enough. The problem is that students never "see" the effect of applying a test input, and really "catch" the bug at fault. This situation is, in a way, similar to training combat engineers in landmine detection, by only presenting the techniques in theory, without showing them a landmine explosion in action. In the absence of an incentive to combine techniques in an effective bug-finding strategy, students are left to dogmatically memorize procedures, not really convinced of their added value. An unfortunate situation in our eyes, now when a high-quality test strategy is exactly what the software industry, permanently haunted by time-to-market pressure, desperately needs.

(2) Testing bug-less software is uninspiring. The second problem is that even in the rare cases when one provides students with real, executable code implementations, these contain zero to few fascinating bugs. On the one hand, this is normal, if we think that real-life software products are developed by competent programmers, with the best intentions, who rarely make "stupid" mistakes. On the other hand, these almost perfect artifacts make students perceive testing as a tedious and unrewarding chore. This is the case in many traditional courses, including our own before the
VU-BugZoo intervention. The hypothesis that raises from this analysis is that an end-to-end testing of executable code, abundantly seeded with the most flabbergasting bugs, will better inspire and motivate students, and eventually remedy their negative attitude towards software testing, boosting their willingness to pursue a future career in the field. In order to check this hypothesis, we recently developed an innovative digital learning platform, called VU-BugZoo [31]. Built upon a repository of standalone and embedded, corrupted executable code, VU-BugZoo engages students in a fascinating, "test-until-it-explodes" bug-hunting experience. The idea to use buggy code in teaching software testing is not new $[11,13]$. However, our platform distinguishes from similar initiatives mainly through its repository of software embedded in miniature smart homes, autonomous cars and trains, and medical devices. Although this software repository is still work in progress, the first version of the standalone-software based platform is already functional. In this paper, we will report on its successful pilot deployment for online assessment of the students enrolled in our software testing course during the COVID-19 pandemic. The experiment which was evaluated through student surveys, consisted of an individual bug-hunting challenge that replaced the written exam. In particular, our experiment aimed to find answers to the following research questions.

$R Q 1$. Is software testing learning more effective when the software under test is a $100 \%$ bug-guarantee executable?

$R Q 2$. Is testing more exciting if one knows that there is a bug inside?

RQ3. Is a dynamic bug-hunting challenge an adequate assessment instrument?

The first two research questions aim to check to what extent we addressed the root causes mentioned previously. The goal of RQ3 is to investigate whether replacing the traditional pen-and-paper exam with an individual challenge in VU-BugZoo is a viable option for a future jeopardized by pandemic-related uncertainties. The surveys show that students liked the intervention, which added in their opinion more fun, excitement and effectiveness to their learning. Moreover, evaluations showed a moderate increase in students' willingness to pursue a future career in testing. The reminder of the paper proceeds as follows. Section 2 describes the design and organizational structure of VU-BugZoo web application. Section 3 describes its deployment for a remote examination and summarizes the students' evaluations and our lessons learned. Section 4 places our contribution in the context of related work. Last, Section 5 outlines our conclusions and future plans.

\section{VU-BUGZOO WEB APPLICATION DESIGN}

\subsection{High-level design}

The core of VU-BugZoo platform is a web application ${ }^{1}$, built around a corpus of fault-seeded executable code. The idea is to practice black-box or white-box testing on these artifacts, and understand the power of different test generation techniques to discover bugs. The final goal is to teach students how to devise a sound test strategy that detects the seeded faults in a shortest time and with a minimum effort. What makes our platform innovative is that it allows an end-to-end dynamic testing of executable code, covering

\footnotetext{
${ }^{1}$ https://dbugit.io/
} 
the entire test process, in contrast to other traditional courses that mainly cover only the test generation step. An assignment consists of a set of correct requirements specifications, and an executable implementation. Let us take as a working example a Body Mass Index (BMI) calculator that based on the height (in meters) and weight of a person (in kilograms), calculates and displays the BMI, defined as:

$$
B M I=\frac{\text { weight }}{\text { height }^{2}}\left[\frac{\mathrm{kg}}{\mathrm{m}^{2}}\right]
$$

The requirements specify in natural language the functionality of the module, its inputs and expected output. For each set of requirements, the buggy artifacts corpus contains one basic variant with a correct implementation, denoted for our example by BMI (0), and a few mutants, each containing a bug injected in the source code, denoted by BMI (1), BMI (2), etc. as shown in Fig. 1. The test techniques currently demonstrated in our platform belong to the category Black-box (selected value, Boundary Value Analysis (BVA), Equivalence Partitioning (EP), Decision tables, Model based Testing (MBT), or combinatorial testing) and White-box (controlflow testing and data-flow testing). The database is extensible, and the teacher can add other techniques and other assignments if needed. The programming languages used to create the executables are currently Python and C. The types of bugs we injected so far are inspired from mutation testing literature and existing bug taxonomies, and are roughly divided in three groups, namely "omitted", "committed" and "wrong" implementation. For example for the BMI calculator assignment, that expects two input parameters, given by the height and weight of a person, a possible bug would be to omit to implement a check for the selected value for height equaling 0 . Another possible bug would be to allow the user to input a wrong number of parameters, for example only one instead of two. Finally, a classical bug could be to use a wrong formula to calculate the BMI. VU-BugZoo platform allows to run an executable for an unlimited number of times, followed by a display of the output for any combination of input parameters. The testing process ends with a submission, containing a selection of test cases and a test report in which students justify their test strategy and present findings. VU-BugZoo has a modular structure, in which more assignments can be combined to create a challenge. A challenge can be seen as a practical exercise in a tutorial, or a remote online exam. Challenges can be used in two modes, as a Student or as a Teacher. A student has access to the challenge and a limited number of assignments for practice. A teacher has access to all the assignments and submissions. A description of the bug that has been injected in each assignment is also accessible by the teacher, allowing for faster and easier grading. Moreover, a teacher also has an administrator role, and can create new challenges, by selecting assignments from the database. The user interface consists of a few pages and buttons, that allow students to login, to execute and submit tests, to see the console output, and finally to compose a test report (see Fig. 2).

\subsection{Low-level design and implementation}

The VU-BugZoo web application for testing standalone software was built in five months using GitLab, an open source end-to-end software development platform with built-in version control, issue tracking, code review, $\mathrm{CI} / \mathrm{CD}$, and many other features that help

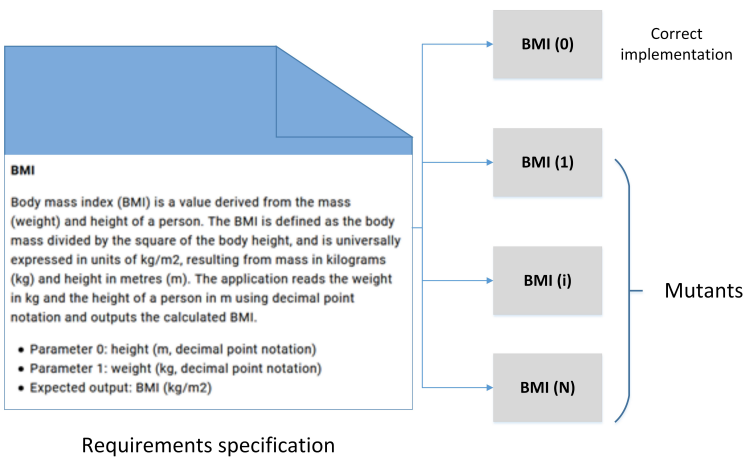

Figure 1: A sample assignment in VU-BugZoo.

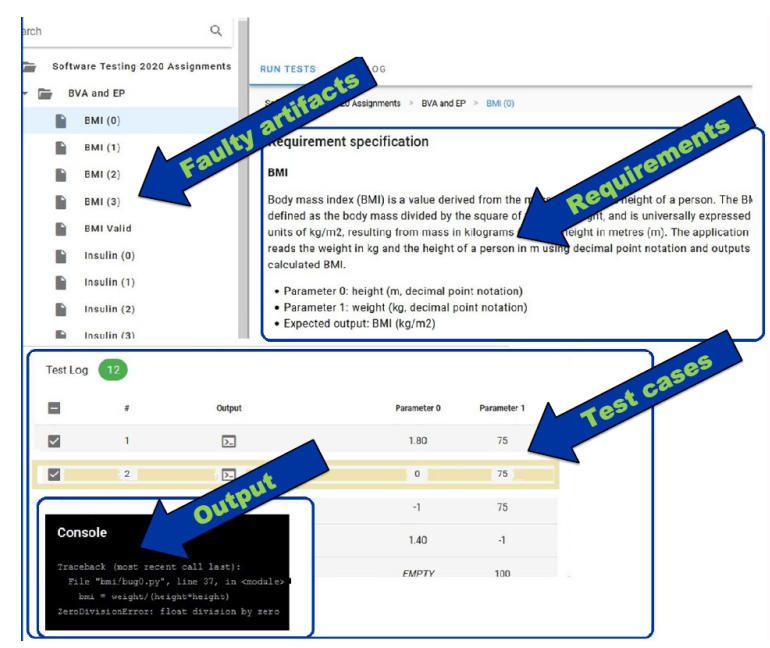

Figure 2: A summary of the main screens in VU-BugZoo interface, showing on the left side the assignments tree, grouped by test technique, and for the particular case of the BMI calculator assignment, (1) the requirements specification, (2) the test log and (3) the output returned to the user for one particular test case (Height $=0$, Weight $=75 \mathrm{~kg}$ ) showing a division by zero error message.

teams improve the cycle time from weeks to minutes, reduce development costs and time to market while increasing productivity [25].

Building the back-end for a robust, software testing learning platform like VU-BugZoo, raised the following challenges.

(1) How to protect the platform from unauthorized access and unintended interactions and overall invalid operations?

(2) How to prevent the machine from crashing anytime an invalid test input forces the code in an assignment to do so? Think here of division by zero in the BMI calculator.

3) How to prevent hackers from exploiting certain types of software bugs and take control over a machine?

4) How to prevent the system from entering an infinite loop? The core back-end functionality of VU-BugZoo was realised using 
Flask, a Python framework suitable for rapid web design and development [24]. The functionality of the back end is split into three main components: core, routes and database. The core component initializes the server, reads and engages all configuration parameters, boots up the database and fills it up with predefined data. The routes component defines the API endpoints that the front end can interact with. Any request from the front end is interpreted and checked by the route's functionalities. These checks ensure that the user who requests the information has the proper permissions to access the data (challenge \#1). The assignments in VU-BugZoo are deployed in a Docker container. The advantage of this solution is twofold. First, it ensures that only the container containing the threatened assignment will crash, and not the machine itself (challenge\#2). Also, the use of Docker containers adds an additional layer of security (challenge \#3). By having the assignment running in a container, a hacker would be contained only to the assignment container itself, instead of the entire machine. Moreover, in order to prevent infinite loops (challenge \#4), a cron-job was implemented, to check and kill any Docker container running for more than 10 minutes. Once an assignment code is executed, the results are stored in the database and the container is removed. This approach allows for previously stored results to be retrieved across users, instead of rerunning the assignment for each identical run of an assignment.

The VU-BugZoo front end is responsible for the interaction with the user, who can be a student or a teacher. The front end functionality was implemented using Vue.js, an open-source JavaScript framework for building fast and robust user interfaces [27]. The advantages of this platform are a clear and well-written documentation, html templating, and well-developed and maintained key tools and libraries needed for every web application development. The user interface was developed using Vuetify, a complete UI framework built on top of Vue.js. This framework uses Google's Material design, with components inspired from the physical world, that create a hierarchy of elements using shadows, shades and shapes, thriving to "reimagine the mediums of paper and ink" [26].

\section{FIRST RESULTS}

\subsection{The BUG_HUNT challenge}

In the Spring of 2020, the course Software Testing unfolded in a completely unexpected and unusual manner, caused by the COVID-19 university lock-down. All written exams requiring physical presence on the campus got cancelled, with instructors being advised to quickly find alternative, remote solutions. Although VU-BugZoo was at that time still under construction, and in the first instance designed only for practice and demonstration, we saw in this context the golden opportunity to prove its innovative concept. For this purpose, we used VU-BugZoo to design a "100\% bug-guarantee" individual challenge, called BUG_HUNT, aimed to replace the written exam. Due to its experimental nature, BUG_HUNT counted for only $25 \%$ of the final grade. Each student got an account in VU-BugZoo and five days to work on their individual challenge. The Bug_HUNT challenge consisted of two assignments, one for black-box testing, with only requirements and an executable available, and one for white-box testing, where code was visible as well. After five days, we closed the challenge and we graded the students' last submissions. We instructed students to submit the following

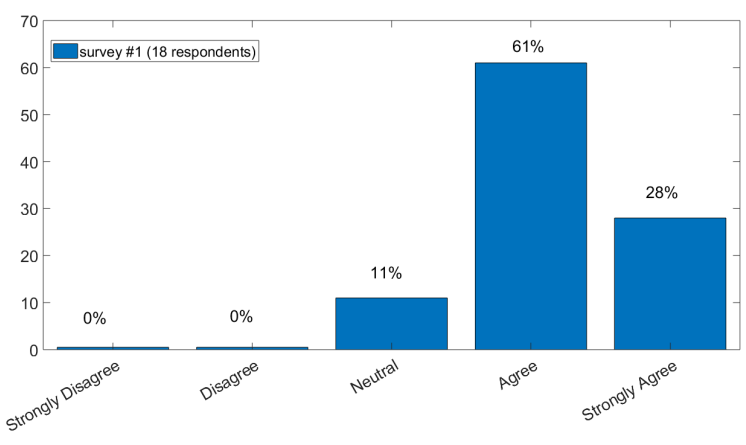

Figure 3: Student's answers to RQ1. Is software testing learning more effective when the software under test is a $\mathbf{1 0 0 \%}$ bug-guarantee executable?

deliverables : (1) all relevant test inputs (stored in VU-BugZoo and visible for the teacher) ; (2) a test report, where they specify and justify their test strategy, in particular which test generation technique they used and why, which assumptions they took, what was the expected output, what did they use as a test oracle, which criteria they used to decide when a test case passed or failed, followed by (3) a description of the bug(s) they found, using a known bug taxonomy.

\subsection{Evaluation and lessons learned}

In the Spring of 2020, 50 graduate students enrolled in the pilot course. We conducted three student surveys to evaluate the VUBugZoo intervention. Survey \#1, answered by 18 students, had only questions specific to the tool. Survey \#2, answered by 14 students, evaluated the entire course and survey \#3, answered by 24 students, has only one question about a future test career. All surveys contained closed questions, using a three-point or five-point Likert scale (Strongly Disagree, Neutral, Agree, Strongly Agree) and a few open questions.

Answers to RQ1: The first encouraging result is that a large majority of the students ( $89 \%$ ) felt that software testing learning was more effective in a tool like VU-BugZoo, as shown in Fig. 3.

Answers to RQ2: We can see in Fig. 4 that even before completing the course, but especially after, $79 \%$ of the students found testing more exciting when knowing that there is a bug inside. Good to mention that students had an objective way to compare, because their previous assignments in this course tested code written by their fellow students, intended to be "bug-free-by-design".

Answers to RQ3: The results of the surveys (see Fig. 5) show that especially after the completion of the course, $86 \%$ of the respondents found a dynamic bug-hunting challenge as an adequate instrument for their software testing skills.

Moreover, we asked students about the emotions they experienced while working on the BUG_HUNT assignment. From their responses we discovered that working in the VU-BugZoo platform unlocked various feelings, mostly positive, such as excitement, curiosity and fun, but also frustration, mainly caused by the imperfection of the first interface prototype, the stress of not finding the bug, or disappointment that the bug was too easy to find 


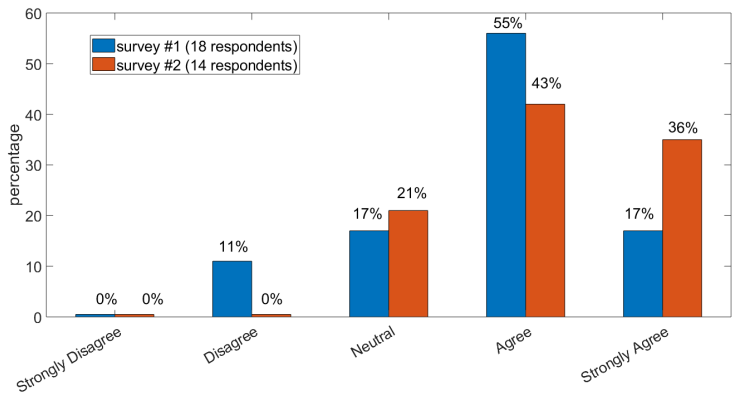

Figure 4: Students' answers to RQ2. Is testing more exciting if one knows that there is a bug inside?

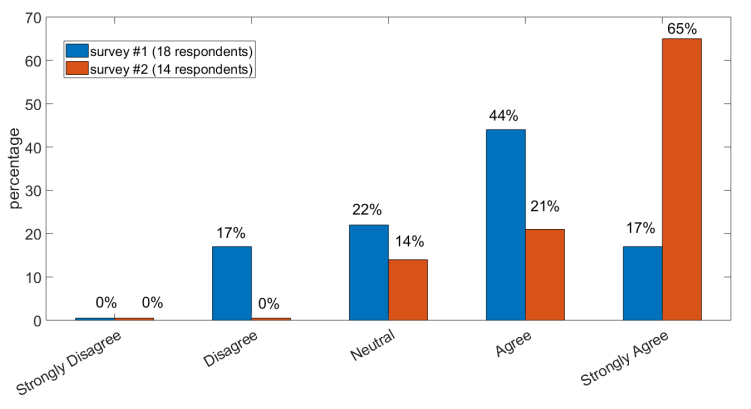

Figure 5: Students' answers to RQ3. Is a dynamic bughunting challenge an adequate assessment instrument?

(see Table 2). Interestingly, one student found testing while knowing that there is a bug inside in contrary, less exciting, because it makes everything more predictable. The main suggestion of students was to equalize the level of bugs difficulty and offer feedback during the practice assignments. We also measured the effect of the BUG_HUNT intervention through a few specific questions, as shown in Table 3. If we compare these results with the situation before the intervention, illustrated back in Table 1, we can see that the enthusiasm towards the topic increased from around $70 \%$ to around $90 \%$. However, when asked about their plans to pursue a future career in software testing, only $38 \%$ from 24 respondents answered with "Yes". This is nevertheless, a bit higher than the $35 \%$ measured in our course in previous years. Moreover the baseline of $35 \%$ was already high, when compared with the average reported elsewhere which is around $20 \%$. The grading time in the new setup was on average 10 minutes per student, which is shorter than the time we normally needed to read and grade a paper-and-pen exam (20-30 minutes). We think that with BUG_HUNT we assessed the students' skills of applying acquired knowledge in practice, while the written exam could better assess their theoretical knowledge. Also, we understood from the evaluations that students would like to have in advance more templates for the test report. Therefore, in the future, we will investigate an automatic system of grading and feedback generation. After grading the submissions, we discovered that almost all students managed to discover the bugs we have seeded. The few ones that were not found were really wicked,
Table 2: Excerpt from students' comments related to BUG_HUNT challenge

\begin{tabular}{|l|}
\hline "I laughed out loud when I found the bug in black box testing. I \\
had a very nice assignment for this and I enjoyed testing it." \\
\hline "I really enjoyed the search for bugs and especially finding \\
them." \\
\hline "The main feeling was excitement when I narrowed down the \\
faulty behavior with a test case." \\
\hline "Excitement, since this method of assessment is more interesting \\
than an exam on paper. Disappointment since the bugs were \\
too obvious." \\
\hline "Wanting to find more bugs then were placed in the assignment, \\
so I guess it can really trigger you to want to test hard! Which \\
is I guess good!" \\
\hline "I didn't feel that excited when I found one, since it was expected \\
in a way." \\
\hline "I was quite enthusiastic about trying all techniques to deter- \\
mine bugs. I hope that future courses have the same setup as \\
this is a more useful evaluation tool for students than an exam." \\
\hline "Enthusiastic - since you can run the program by just passing \\
the arguments and let the program execute it one after one." \\
\hline "Excitement when I found the bug." \\
\hline "I was stressed a bit because I was not able to spot the bug in the \\
white-box testing part before making the tactic for generating \\
test cases." \\
\hline "A bit more complicated programs would seem possible (in the \\
case of white box)." \\
\hline "The only change is that the exam got replaced with the BUG- \\
HUNT assignment, which is a big plus in my opinion." \\
\hline "BUG_HUNT can be more difficult. In my opinion they were \\
very straight forward and relatively simple to find." \\
\hline "VU-BugZoo should definitely be a part of the course until the \\
end of time." \\
\hline
\end{tabular}

difficult to find bugs. This is another point of concern learned from our experiment - to search for more challenging bugs to inject, while keeping a fair load balance across the assignments. Interestingly, the students were very excited to detect even more bugs than we initially intended to inject. This is normal, as it confirms that developers are also humans and humans make involuntary mistakes. This demonstrates that that intentionally injected bugs can also lead to side-effects and unintentional buggy behaviour elsewhere. Moreover, we noticed that some students focused more on the description of the bugs they found (the "trophies") than on the test strategy that led to finding the bugs (the "weapons"). Also, some submitted all the test cases they have ever tried, creating an overhead, without any test minimization effort. This means we partially missed our goal to teach students how to design a good strategy to detect bugs. In the future, we will have to emphasize more strongly the importance of a sound test strategy.

\subsection{Threats to validity}

The most important threat to internal validity is the low student response to the surveys. Also, as we trusted our students, we did 
Table 3: Excerpt from the students evaluations after the BUG_HUNT intervention (survey \#2 and \#3).

\begin{tabular}{|l|c|c|c|c|c|c|}
\hline Question & - & +- & + & $\mathrm{N}$ & Agree & Delta \\
\hline Interesting course & 0 & 1 & 13 & 14 & $93 \%$ & $+26 \%$ \\
\hline $\begin{array}{l}\text { The relevance to the } \\
\text { programme was clear }\end{array}$ & 0 & 2 & 12 & 14 & $86 \%$ & $+10 \%$ \\
\hline $\begin{array}{l}\text { I would like working } \\
\text { in the software testing } \\
\text { field }\end{array}$ & 6 & 9 & 9 & 24 & $38 \%$ & $+3 \%$ \\
\hline
\end{tabular}

not implement any mechanisms for fraud detection or plagiarism during the BUG_HUNT challenge. Next year, we will have to take into consideration that the already injected bugs might not be so new anymore and will be easier to disclose. We will solve this threat by devising new assignments, featuring new, more fascinating bugs. Also, the student population we interviewed every year is different. This is why in Table I we combined the evaluations collected from two years, 2018 and 2019, in order to obtain a more reliable baseline.

A threat to the external validity of our experiment is that the users in our pilot were only graduate students without previous experience in software testing. In order to mitigate this threat, we will seek contact with industry partners and evaluate our tool with more experienced testers. Since these are preliminary results, a lot still has to be done, such as for example a usability study.

\section{RELATED WORK}

Bug repositories containing code snippets in Java, $\mathrm{C} / \mathrm{C}++$ and $\mathrm{C \#}$ exist already, serving mainly to enable researchers to perform controlled experimentation and replications, or for automatic program repair. Examples are the Software-artifact Infrastructure Repository (SIR) [12], Defects4J [14] and the more recent BugZoo [35]. However, all these repositories contain exclusively standalone software and were not primarily intended for software testing teaching. Our platform will contain in the future additional examples of software tightly integrated with hardware in Internet-of-Things (IoT) devices. This mix is, as far as we are aware, unique.

A few courses also use bug-contaminated code for assessing students' testing skills. For example, a bug hunting game to make software testing more exciting was reported by Cleggs et al in [6] These authors designed Code Defenders, a multiplayer game where attackers create mutants and defenders need to make test cases that detect them. Our platform is also a game, but a single player one, where we are the attackers who created apriori the mutants and students are in fact the defenders. However, our paper also contains an evaluation of the approach, in addition to only a description of the platform. Aaltonnen [1] and deMilo [11] used aposteriori mutation testing to assess the test cases after they have been designed by students, in order to motivate them to continue testing and search for more bugs. Our platform apriori created mutated code instead. A similar approach was reported by Smith et al., in [32]. The authors developed an interactive learning system, targeted at introductory students that aims to teach how to design good test cases. As a feedback, students get to see as an incentive, mutated "buggy" code that is still not detected by their test cases. The most closely-related work is the web-based tutorial built by Elbaum et al, called BugHunt
[13]. It consists of lessons and instructions, where students have to devise tests using a particular test technique, and submit them to the server which executes them. As a reward, students get to see the bug they found shown in a bug jar. We don't have a bug jar yet, but intend to implement it in the future. The main difference with respect to all these contributions is that in our case, the pilot course is dedicated to software testing and targets graduate, instead of CS1/CS2 students. Moreover, our grading is based not so much on the act of bug finding itself, but on the quality of the test strategy, and on the accuracy of the bugs diagnosis. On the other hand, we are aware that our first version is still quite basic, using manual instead of automatic grading, and does not have any feedback mechanism implemented yet. Also, adding new assignments through the user interface is not yet possible. Fault injection is a technique to assess the dependability of systems by seeding failure modes in hardware or in software $[19,20,36]$. When designing a fault injection experiment, the most challenging decision concerns which bugs to inject [14]. Up to now, for our platform we used the most common operators used in mutation testing [2,21,22] and the most common types of errors specified in the well-known Knuth's [15] and Beizer's bug taxonomies [3].

\section{CONCLUSIONS AND FUTURE WORK}

We presented VU-BugZoo, a new learning platform developed to increase student's motivation in a software testing classroom. Its first deployment showed promising preliminary results, reflected in a higher excitement and learning effectiveness, together with a positive shift in students' general attitude towards software testing. Moreover, the online bug-hunting challenge was perceived by students as an adequate replacement for a pen-and-paper traditional exam, very welcome during the COVID-19 pandemic. Finally, our intervention led to a slight increase in students' willingness to take up a future career in the field. The latter result confirms that winning souls for the software testing field remains a tough mission.

Although one realizes that software testing is much more than just bug-hunting, we strongly believe that the proposed approach is very effective in increasing the motivation of the very beginners. Therefore, we will continue to develop the tool and use it in our teaching. In the future, we will be extending the corpus of corrupted artifacts with both synthetic and historical, representative, wellhidden bugs. Moreover, we will extend the tool's functionality with automatic grading and feedback generation. Finally, we will investigate ways to make the tool available to interested communities, both in academia and industry.

\section{ACKNOWLEDGMENT}

This project is funded by the NRO, The Netherlands Initiative for Education Research, as part of a Comenius Teaching Fellow grant. The authors would like to thank the VU Computer Science department and Stefan Schlobach in particular, for co-financing the project; Herbert Bos and Wan Fokkink for their useful suggestions and encouragements; finally, all of the CS students who participated in the software testing course, and in particular the 2020 cohort, who endured with stoicism our pilot experiment, and blessed us with their precious suggestions and encouragements. 


\section{REFERENCES}

[1] Kalle Aaltonen, Petri Ihantola, and Otto Seppälä. 2010. Mutation Analysis vs. Code Coverage in Automated Assessment of Students' Testing Skills. In Proceedings of the ACM International Conference Companion on Object Oriented Programming Systems Languages and Applications Companion (Reno/Tahoe, Nevada, USA) (OOPSLA '10). Association for Computing Machinery, New York, NY, USA, 153-160. https://doi.org/10.1145/1869542.1869567

[2] James H Andrews, Lionel C Briand, and Yvan Labiche. 2005. Is mutation an appropriate tool for testing experiments?[software testing]. In Proceedings. 27th International Conference on Software Engineering, 2005. ICSE 2005. 402-411.

[3] Boris Beizer. 1990. Software Testing Techniques.

[4] L. F. Capretz, S. Basri, Maythem Adili, and Aamir Amin. 2020. What Malaysian Software Students Think about Testing? Proceedings of the IEEE/ACM 42nd International Conference on Software Engineering Workshops (2020).

[5] Luiz Fernando Capretz, Pradeep Waychal, Jingdong Jia, Daniel Varona, and Yadira Lizama. 2019. Studies on the Software Testing Profession. In Proceedings of the 41st International Conference on Software Engineering: Companion Proceedings. 262-263. https://doi.org/10.1109/ICSE-Companion.2019.00105

[6] Benjamin S. Clegg, José Miguel Rojas, and Gordon Fraser. 2017. Teaching Software Testing Concepts Using a Mutation Testing Game (ICSE-SEET '17). IEEE Press, 33-36. https://doi.org/10.1109/ICSE-SEET.2017.1

[7] Lee Copeland. 2004. A Practitioner's Guide to Software Test Design. Artech House.

[8] P. Coy and M. Lewyn. 1990. The day that every phone seemed off the hook Business Week (1990), 39-40.

[9] Anca Deak, Tor Stålhane, and Guttorm Sindre. 2016. Challenges and Strategies for Motivating Software Testing Personnel. Inf. Softw. Technol. (2016), 1-15. https://doi.org/10.1016/j.infsof.2016.01.002

[10] Anca Deak, Tor Stålhane, and Daniela Cruzes. 2013. Factors Influencing the Choice of a Career in Software Testing among Norwegian Students. IASTED Multiconferences - Proceedings of the IASTED International Conference on Software Engineering, SE 2013 (03 2013). https://doi.org/10.2316/P.2013.796-032

[11] Richard A DeMillo, Richard J Lipton, and Frederick G Sayward. 1978. Hints on test data selection: Help for the practicing programmer. Computer 11, 4 (1978), $34-41$.

[12] H. Do, S. Elbaum, and G. Rothermel. 2005. Supporting Controlled Experimentation with Testing Techniques: An Infrastructure and its Potential Impact. Empirical Software Engineering 10 (2005), 405-435.

[13] S. Elbaum, S. Person, J. Dokulil, and M. Jorde. 2007. Bug Hunt: Making Early Software Testing Lessons Engaging and Affordable. In 29th International Conference on Software Engineering (ICSE'07). 688-697. https://doi.org/10.1109/ICSE.2007.23

[14] René Just, Darioush Jalali, and Michael D. Ernst. 2014. Defects4J: A Database of Existing Faults to Enable Controlled Testing Studies for Java Programs. In Proceedings of the 2014 International Symposium on Software Testing and Analysis. 437-440. https://doi.org/10.1145/2610384.2628055

[15] Donald E Knuth. 1989. The errors of TEX. Software: Practice and Experience 19, 7 (1989), 607-685.

[16] N. G. Leveson and C. S. Turner. 1993. An investigation of the Therac-25 accidents. Computer 26, 7 (1993), 18-41.

[17] Yadira Lizama-Mué, Daniel Varona, Pradeep Waychal, and Luiz Capretz. 2020 The Unpopularity of the Software Tester Role Among Software Practitioners: A Case Study. 185-197. https://doi.org/10.1007/978-3-030-36518-97
[18] Aditya Mathur. 2008. Foundations of Software Testing. Addison-Wesley.

[19] Mei-Chen Hsueh, T. K. Tsai, and R. K. Iyer. 1997. Fault injection techniques and tools. Computer 30, 4 (1997), 75-82.

[20] Roberto Natella, Domenico Cotroneo, and Henrique S. Madeira. 2016. Assessing Dependability with Software Fault Injection: A Survey. ACM Comput. Surv. 48, 3 , Article 44 (Feb. 2016). https://doi.org/10.1145/2841425

[21] Jefferson Offut. 2011. A mutation carol: Past, present and future. Information and Software Technology 53, 10 (2011), 1098 - 1107. https://doi.org/10.1016/j.infsof. 2011.03.007

[22] A Jefferson Offutt, Ammei Lee, Gregg Rothermel, Roland H Untch, and Christian Zapf. 1996. An experimental determination of sufficient mutant operators. $A C M$ Transactions on Software Engineering and Methodology (TOSEM) 5, 2 (1996), 99118

[23] Online. [n.d.]. Boeing 737 MAX: What's Happened After the 2 Deadly Crashes. https://www.nytimes.com/interactive/2019/business/boeing-737crashes.html. Accessed: 2020-10-10.

[24] Online. [n.d.]. Flask. https://flask.palletsprojects.com. Accessed: 2020-10-10

[25] Online. [n.d.]. Gitlab. https://about.gitlab.com/. Accessed: 2020-10-10.

[26] Online. [n.d.]. Material Design. https://material.io/design/. Accessed: 2020-10-10.

[27] Online. [n.d.]. Vue.js-The Progressive JavaScript Framework. https://vuejs.org/. Accessed: 2020-10-10.

[28] Mauro Pezzè and Michal Young. 2007. Software testing and analysis - process, principles and techniques. Wiley.

[29] Hina Shah and Mary Jean Harrold. 2010. Studying Human and Social Aspects of Testing in a Service-Based Software Company: Case Study. In Proceedings of the 2010 ICSE Workshop on Cooperative and Human Aspects of Software Engineering.
Association for Computing Machinery, New York, NY, USA, 102-108. https: //doi.org/10.1145/1833310.1833327

[30] Natalia Silvis-Cividjian. 2018. A Safety-Aware, Systems-Based Approach to Teaching Software Testing. In Proceedings of the 23rd Annual ACM Conference on Innovation and Technology in Computer Science Education. 314-319. https: //doi.org/10.1145/3197091.3197096

[31] Natalia Silvis-Cividjian, Rob Limburg, Niels Althuisius, Emil Apostolov, Viktor Bonev, Robert Jansma, Glenn Visser, and Marc Went. 2020. VU-BugZoo: A Persuasive Platform for Teaching Software Testing. In Proceedings of the 2020 ACM Conference on Innovation and Technology in Computer Science Education (Trondheim, Norway) (ITiCSE '20). 553. https://doi.org/10.1145/3341525.3393975

[32] Rebecca Smith, Terry Tang, Joe Warren, and Scott Rixner. 2017. An Automated System for Interactively Learning Software Testing. In Proceedings of the 2017 ACM Conference on Innovation and Technology in Computer Science Education (Bologna, Italy) (ITiCSE '17). Association for Computing Machinery, New York, NY, USA, 98-103. https://doi.org/10.1145/3059009.3059022

[33] Ian Sommerville. 2010. Software Engineering (9 ed.). Addison-Wesley.

[34] Standard. 2013. ISO/IEC/IEEE 29119-3:2013, Part 3: Test documentation.

[35] Christopher Steven Timperley, Susan Stepney, and Claire Le Goues. 2018. BugZoo: A Platform for Studying Software Bugs. In Proceedings of the 40th International Conference on Software Engineering: Companion Proceeedings. 446-447. https: //doi.org/10.1145/3183440.3195050

[36] J. Voas. 1997. Software fault injection: growing 'safer' systems. In 1997 IEEE Aerospace Conference, Vol. 2. 551-561 vol.2.

[37] P. Waychal and L. F. Capretz. 2016. Why a Testing Career Is Not the First Choice of Engineers. ArXiv abs/1612.00734 (2016). 